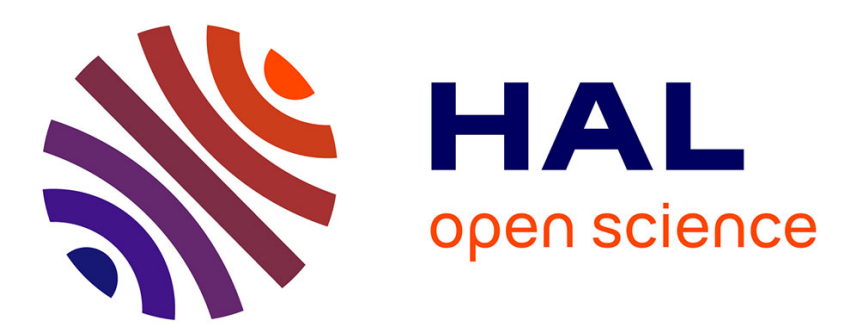

\title{
A comparison of a one-dimensional finite element method and the transfer matrix method for the computation of wind music instrument impedance
}

Robin Tournemenne, Juliette Chabassier

\section{- To cite this version:}

Robin Tournemenne, Juliette Chabassier. A comparison of a one-dimensional finite element method and the transfer matrix method for the computation of wind music instrument impedance. Acta Acustica united with Acustica, 2019, 105 (5), pp.838. 10.3813/AAA.919364 . hal-01963674v2

\section{HAL Id: hal-01963674 \\ https://hal.inria.fr/hal-01963674v2}

Submitted on 24 Oct 2019

HAL is a multi-disciplinary open access archive for the deposit and dissemination of scientific research documents, whether they are published or not. The documents may come from teaching and research institutions in France or abroad, or from public or private research centers.
L'archive ouverte pluridisciplinaire HAL, est destinée au dépôt et à la diffusion de documents scientifiques de niveau recherche, publiés ou non, émanant des établissements d'enseignement et de recherche français ou étrangers, des laboratoires publics ou privés. 


\title{
A comparison of a one-dimensional finite element method and the transfer matrix method for the computation of wind music instrument impedance
}

\author{
Robin Tournemenne $^{1)}$, Juliette Chabassier ${ }^{1)}$ \\ 1) Magique 3D Team, Inria Bordeaux Sud Ouest, 200 avenue de la vieille tour, \\ 33405 Talence Cedex, France, firstname.lastname@inria.fr
}

\section{Summary}

2 This work presents a computation tool for the calculation of wind instrument input impedance in the context of linear planar wave propagation with viscothermal losses. The originality of the approach lies in the usage of a specific and simple 1D finite element method (FEM). The popular Transfer Matrix Method (TMM) is also recalled and a seamless formulation is proposed which unifies the cases cylinders vs. cones. Visco-thermal losses, which are natural dissipation in the system, are not exactly taken into account by this method when arbitrary shapes are considered. The introduction of an equivalent radius leads to an approximation that we quantify using the FEM method. The equation actually solved by the TMM in this case is exhibited. The accuracy of the two methods (FEM and TMM) and the associated computation times are assessed and compared. Although the TMM is more efficient in lossless cases and for lossy cylinders, the FEM is shown to be more efficient when targeting a specific precision in the realistic case of a lossy trumpet. Some additional features also exhibit the robustness and flexibility of the FEM over the TMM. All the results of this article are computed using the opensource python toolbox OpenWind.

\section{Introduction}

27 The input impedance of wind instruments is defined as its frequency dependent linear response to an input excitation. This physical quantity is of considerable advantage in understanding the instrument's playing quality, and eventually its musical behavior Campbell(2004), Chaigne and Kergomard(2016)]. The impedance is used for various purposes, such as the analysis of the instrument's playing properties, the synthesis of their sounds and the design of their shape. Indeed, many studies try to correlate the impedance features to the instrument actual intonation, stability, tone Backus(1976), Braden et al.(2009), Campbell(2004). Many syn- thesis methods rely on the input impedance knowledge to produce realistic sounds [Silva et al.(2014)], in order to assess the quality of the physical model, or to provide musicians with virtual instruments. Wind instrument design is the goal of many current initiatives, which try to either reconstruct bores, solve inverse problems based on their measured input impedance $[\operatorname{Kausel}(2001)]$, improve existing instruments Tournemenne et al.(2017) or even develop new instruments Buys et al.(2017) to fulfill the aspirations of musicians.

On the one hand, since the pioneering work of Webster Webster(1947)], many methods can measure the input impedance with varying precision and frequency range Le Roux et al.(2008), Caussé et al.(1984), Sharp et al.(2011). hand, physical models associated with computation methods can be used to calculate the input impedance. The current reference computation method is the transfer matrix method (TMM), which has been used in the context of wind instruments for more than 40 years Plitnik and Strong(1979), Mapes-Riordan(1993)]. The underlying physical model can assume plane or spherical wave propagation in the pipe, mono or multi-modal propagation, viscothermal losses at the pipe walls and a radiation impedance at the pipe output, etc.

The objective of this paper is to propose a new method for the computation of the input impedance, which could noticeably facilitate and broaden numerical instrument design approaches. It is not our purpose in this article to discuss the physical model and especially the validity of the underlying physical assumptions. Although this topic is of great interest, and must rely on precise simulation / measurement comparisons, the present work only focuses on technical aspects of the impedance computation. The methodology is here presented in the simplest possible realistic acoustical case, but the present article will serve as a basis to consider more general physical models in the future. We will present a new computation approach based on a one-dimensional finite element method used on the Telegraph equations with viscothermal losses. No- 
tice first that, compared to the TMM, the proposed approach is therefore simply another way of solving the same equations. Notice also that the objective is not to solve the acoustical equations in 3D Lefebvre and Scavone(2012), nor the Navier-Stokes equations in 3D [Giordano(2014)]. The method proposed in this paper is close to finite difference methods Bilbao(2009), van den Doel and Ascher(2008)], even if it is used here in the time-harmonic context.

This article goes in pair with an open-source Python 3 toolbox, Openwind OpenWInD, that can be freely downloaded and used to undertake numerical experiments. After introducing the physical context in Section 2, the practical aspects of this numerical method (FEM) are first covered in Section 3, then the current reference method, the Transfer Matrix Method, is presented and its limits considering viscothermal losses are exhibited in Section 4. A thorough validation is made in order to assess the precision and performance brought by this one-dimensional finite element implementation in Section 5. The TMM can only approximate the solution when visco-thermal losses are considered for arbitrary shapes. We study the related error using the introduced FEM in Section 6. Finally, computation times and several useful features of the FEM are presented (Section 6.3) before concluding.

\section{Physics-based model}

Consider an axisymmetric pipe occupying a domain $\Omega \subset \mathbb{R}^{3}=(O x, O y, O z)$ of slowly varying cross section $S$ and rigid walls developing along the $x$ axis, filled with air, see Figure 1 .

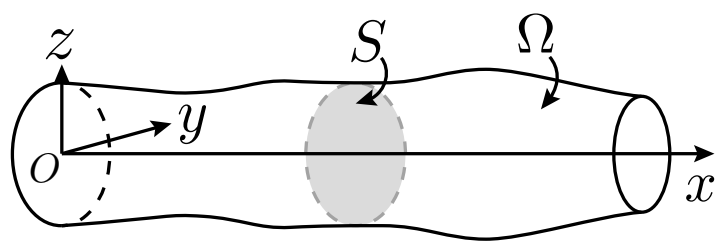

Figure 1: Definition of the space variables. $S$ is the slowly varying section of the axisymmetric pipe.

The acoustic pressure $p(x, y, z, t)$ and the threedimensional flow $u(x, y, z, t)$ can be considered as the solution to Navier-Stokes three-dimensional equations which induce an undue computational burden in the context where only the wave propagating phenomena are of interest. Following the simplifications of Kirchhoff's theory regarding visco-thermal losses near the pipe walls [Kirchhoff(1868), Zwikker and Kosten(1949), Chaigne and Kergomard(2016)], the pressure can be considered as constant in the sections orthogonal to the $x$-axis, the orthogonal components of the
Sound velocity: $c=331.45 \sqrt{T / T_{0}} \mathrm{~m} \mathrm{~s}^{-1}$

Density: $\rho=1.2929 T_{0} / T \mathrm{~kg} \mathrm{~m}^{-3}$

Viscosity: $\mu=1.708 \mathrm{e}-5(1+0.0029 t) \mathrm{kg} \mathrm{m}^{-1} \mathrm{~s}^{-1}$

Thermal conductivity: $\kappa=5.77 \mathrm{e}-3(1+0.0033 t) \mathrm{Cal} /\left(\mathrm{ms}{ }^{\circ} \mathrm{C}\right)$

Spec. heat with constant p.: $C_{p}=240 \mathrm{Cal} /\left(\mathrm{kg}{ }^{\circ} \mathrm{C}\right)$

Ratio of specific heats: $\gamma=1.402$

Table 1: Numerical values Chaigne and Kergomard(2016) of air constants used in the model. $t$ is the temperature in Celsius, and $T$ the absolute temperature with $T_{0}=273.15 \mathrm{~K}$.

three-dimensional flow can be neglected in the equations while the axial component can be considered as axisymmetric with an analytic expression of its radial dependency. Finally, we seek in the frequency domain $\hat{p}(x, \omega)$ the acoustic pressure ${ }^{1}$ and $\hat{u}(x, \omega)$ the volume flow, such that the one-dimensional interior equations read, for all position $x \in[0, L]$ and angular frequency $\omega \in\left[\omega_{\min }, \omega_{\max }\right]$,

$$
\left\{\begin{array}{c}
Z_{v}(\omega, x) \hat{u}+\frac{\mathrm{d} \hat{p}}{\mathrm{~d} x}=0, \\
Y_{t}(\omega, x) \hat{p}+\frac{\mathrm{d} \hat{u}}{\mathrm{~d} x}=0,
\end{array}\right.
$$

$$
\text { (22) }\left\{\begin{array}{c}
Z_{v}(\omega, x)=\frac{j \omega \rho}{S(x)}\left[1-\mathcal{J}\left(k_{v}(\omega) R(x)\right)\right]^{-1}, \\
Y_{t}(\omega, x)=\frac{j \omega S(x)}{\rho c^{2}}\left[1+(\gamma-1) \mathcal{J}\left(k_{t}(\omega) R(x)\right)\right], \\
k_{v}(\omega)=\sqrt{j \omega \frac{\rho}{\mu}}, \quad k_{t}(\omega)=\sqrt{j \omega \rho \frac{C_{p}}{\kappa}},
\end{array}\right.
$$

where $R$ is the section radius, $S=\pi R^{2}$ is the section area, Table 1 describes the air constants, and we introduce the function $\mathcal{J}$ of a complex variable, which models the dissipative terms, as

$$
\mathcal{J}(z)=\frac{2}{z} \frac{J_{1}(z)}{J_{0}(z)}, \quad \forall z \in \mathbb{C},
$$

where $J_{0}$ and $J_{1}$ are the Bessel functions of the first kind. The subscripts $v$ and $t$ respectively stand for viscous and thermal dissipative phenomena.

Furthermore, if the dissipative terms are neglected ( $\mathcal{J}$ function set to zero in the equations), the classical horn equations describing plane wave propagation in an axisymmetric lossless pipe can be retrieved from an asymptotic analysis from Euler's equations in a pipe with a slowly varying section Rienstra(2005). For convenience, we will use the names lossy model for system (1), and lossless model when $\mathcal{J}$ is set to zero in system (1).

Two boundary conditions complete the problem: at the bell $x=L$, we impose a radiation impedance condition

\footnotetext{
${ }^{1}$ variables with a hat $(\hat{\imath})$ denote the time-domain Fourier transform of the unknown
} - 
Rabiner and Schafer(1978), Dalmont et al.(2001). Chaigne and Kergomard(2016) :

$$
\frac{\hat{p}(L, \omega)}{\hat{u}(L, \omega)}=Z_{R}(\omega)
$$

and at the input of the pipe, we impose $\hat{u}(0, \omega)=$ $\lambda(\omega)$, where $\lambda(\omega)$ will be a source term for the system. Since all the considered equations are linear, we can consider without loss of generality $\lambda(\omega) \equiv 1$. In this article, we are interested in computing the input impedance

$$
Z(\omega):=\frac{\hat{p}(0, \omega)}{\hat{u}(0, \omega)}=\hat{p}(0, \omega)
$$

Finally, the considered problem is the following: compute

$$
\left\{\begin{array}{cl}
\{(\omega)=\hat{p}(0, \omega), & \text { where } \\
\begin{cases}Z_{v}(\omega, x) \hat{u}+\frac{\mathrm{d} \hat{p}}{\mathrm{~d} x}=0, & \\
Y_{t}(\omega, x) \hat{p}+\frac{\mathrm{d} \hat{u}}{\mathrm{~d} x}=0, & \\
\hat{u}(0, \omega)=1, & \\
\frac{\hat{p}(L, \omega)}{\hat{u}(L, \omega)}=Z_{R}(\omega) . & \end{cases}
\end{array}\right.
$$

In the subsequent sections, we are interested in possible methods to solve system (7). We will first present the Finite Element Method and then the Transfer Matrix Method.

\section{$3 \quad$ Finite element method}

The finite element method (FEM) relies on a variational formulation of the entire system in usual infinite dimensional Sobolev spaces [Brezis(2011)], followed by the definition of finite dimensional spaces in which we seek numerically the solution. Recall that the Sobolev spaces $L^{2}$ and $H^{1}$ can be physically interpreted as $f \in L^{2}([0, L])$ if $f$ is squared integrable on $[0, L]$ and $f \in H^{1}([0, L])$ if its gradient is squared integrable. For first order formulations as the one of system (7) (flow / pressure), the theory Courant and Hilbert(1965), Cohen (2000) points towards the possible following framework. Find $\hat{p}_{h} \in V_{h} \subset H^{1}([0, L]), \hat{u}_{h} \in W_{h} \subset L^{2}([0, L])$, such that for all $q_{h} \in V_{h}, w_{h} \in W_{h}$,

$$
\left\{\begin{array}{c}
\int_{0}^{L} \frac{j \omega \rho}{S}\left[1-\mathcal{J}\left(k_{v}(\omega) R\right)\right]^{-1} \hat{u}_{h} \overline{w_{h}} \\
+\int_{0}^{L} \frac{\mathrm{d} \hat{p}_{h}}{\mathrm{~d} x} \overline{w_{h}}=0 \\
\int_{0}^{L} \frac{j \omega S}{\rho c^{2}}\left[1+(\gamma-1) \mathcal{J}\left(k_{t}(\omega) R\right)\right] \hat{p}_{h} \overline{q_{h}}-\int_{0}^{L} \frac{\mathrm{d} \overline{q_{h}}}{\mathrm{~d} x} \hat{u}_{h} \\
-\overline{q_{h}}(0) \lambda(\omega)+\frac{1}{Z_{R}(\omega)} \hat{p}_{h}(L) \overline{q_{h}}(L)=0(8 \mathrm{~b})
\end{array}\right.
$$

where by-parts integrations of Equations (7a have been performed, followed by the use of the boundary conditions to weakly give a value to $\hat{u}_{h}(0)$ and $\hat{u}_{h}(L)$. The complex conjugate of $z$ is noted $\bar{z}$. Note that other choices of by-part integrations are possible, associated with other choices of functional spaces. The type of boundary conditions and source regularity usually guide this choice. In practice, we have chosen to use standard Lagrange finite elements, hence to define the spaces $V_{h}$ and $W_{h}$ as follows. Other choices are possible and impact the properties of the method. The instrument is discretized into $N$ elements $\left\{K_{j}\right\}_{j}$, delimited by $N+1$ nodes that constitute the mesh. On each element $K_{j}$ we consider $r+1$ interior degrees of freedom called $\left\{\xi_{j, p}\right\}_{1 \leq p \leq r+1}$.

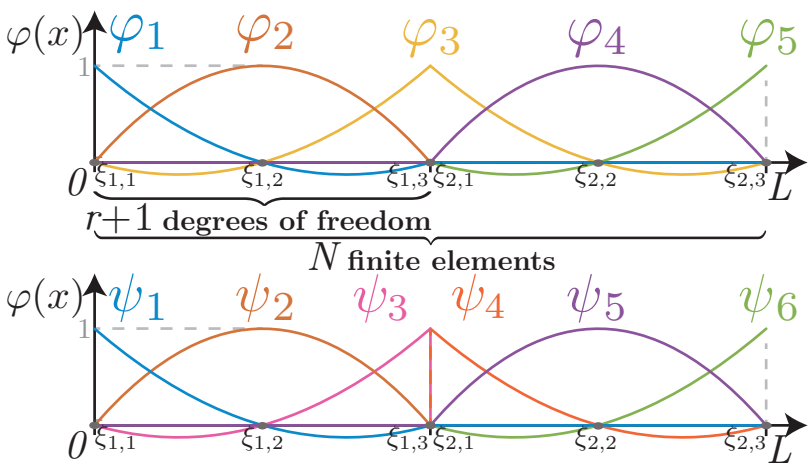

Figure 2: Basis functions with respect to $x$ on a 2elements mesh of $[0, L]$. Top : second order basis function $\left\{\varphi_{i}\right\}_{1 \leq i \leq 5}$ of $V_{h}$. Bottom : second order basis function $\left.\left\{\psi_{i}\right\}_{1 \leq i \leq 6}\right)$ of $W_{h}$. (colors online)

The finite dimensional spaces $V_{h}$ and $W_{h}$ are spanned by the nodal bases $\left\{\varphi_{i}\right\}_{1 \leq i \leq N_{H^{1}}}$ and $\left\{\psi_{j}\right\}_{1 \leq j \leq N_{L^{2}}}$ of piecewise polynomial functions of degree $r$ (see an example of order 2 in Figure 2), which defines the order of the FEM. Consequently, the numerical solutions representing the pressure $\hat{p}_{h}$ and volume flow $\hat{u}_{h}$ are linear combinations of the basis functions $\left\{\varphi_{i}\right\}_{1 \leq i \leq N_{H^{1}}}$ and $\left\{\psi_{j}\right\}_{1 \leq j \leq N_{L^{2}}}$ respectively. In some communities, the basis functions are called shape functions. They are interpolation Lagrange polynomials (drawn Figure 2 associated to the concatenation of all the degrees of freedom of all the elements, where the nodes separating two elements are duplicated for $W_{h}$ but not for $V_{h}$. Consequently, the basis functions of $V_{h}$ are continuous while the ones of $W_{h}$ present a discontinuity at the edges of the elements. This follows the conformal nature of the approximation, namely $V_{h} \subset H^{1}([0, L])$ and $W_{h} \subset$ $L^{2}([0, L])$. Moreover, $N_{H^{1}}<N_{L^{2}}$ as soon as the mesh is composed of more than two elements. Finally, the integral terms in Equations (8) are evaluated through a quadrature procedure Quarteroni et al.(2007). Although a high order quadrature formula could be employed to ensure exact integration, we have chosen to follow the condensation procedure (also named mass- 
lumping procedure) of spectral high order finite elements Cohen(2004) $]^{2}$. This technique is divided into two joint steps: using the same points for the quadrature and the interpolation which leads to a diagonal mass matrix (condensation), and choosing as interpolation points the Gauss-Lobatto points which prevents accuracy loss of the condensation method and improves the global matrix conditioning. Approximate integrals that come from this procedure will be denoted $f$.

Since system (8) stands for every $w_{h} \in W_{h}$ and $q_{h} \in V_{h}$, it is equivalent to state that it stands for every basis vector of $W_{h}$ and $V_{h}$. Besides, we abusively still denote $\hat{u}_{h}$ (resp. $\hat{p}_{h}$ ) for the coordinates of $\hat{u}_{h}$ (resp. $\hat{p}_{h}$ ) in the basis $\left\{\varphi_{i}\right\}_{1 \leq i \leq N_{H^{1}}}$ (resp. $\left\{\psi_{j}\right\}_{1 \leq j \leq N_{L^{2}}}$ ). Consequently, the discrete formulation equivalently takes the matrix form

$$
\left\{\begin{array}{c}
j \omega M_{h}^{L^{2}} \hat{u}_{h}+j \omega N_{h}^{L^{2}}(\omega) \hat{u}_{h}-B_{h} \hat{p}_{h}=0 \\
j \omega M_{h}^{H^{1}} \hat{p}_{h}+j \omega N_{h}^{H^{1}}(\omega) \hat{p}_{h}+\frac{1}{Z_{R}(\omega)} \Sigma_{h} \hat{p}_{h} \\
+B_{h}^{*} \hat{u}_{h}-E_{h}=0
\end{array}\right.
$$

where $*$ designates the adjoint and

$$
\begin{aligned}
& \left(M_{h}^{L^{2}}\right)_{i, j}=f_{0}^{L} \frac{\rho}{S} \psi_{i} \psi_{j},\left(M_{h}^{H^{1}}\right)_{i, j}=f_{0}^{L} \frac{S}{\rho c^{2}} \varphi_{i} \varphi_{j}, \\
& \left(N_{h}^{L^{2}}\right)_{i, j}(\omega)=f_{0}^{L} \frac{\rho}{S} \frac{\mathcal{J}\left(k_{v}(\omega) R\right)}{1-\mathcal{J}\left(k_{v}(\omega) R\right)} \psi_{i} \psi_{j}, \\
& \left(N_{h}^{H^{1}}\right)_{i, j}(\omega)=f_{0}^{L} \frac{S}{\rho c^{2}}(\gamma-1) \mathcal{J}\left(k_{t}(\omega) R\right) \varphi_{i} \varphi_{j}, \\
& \left(B_{h}\right)_{i, j}=-f_{0}^{L} \psi_{i} \frac{d \varphi_{j}}{d x}, \quad\left(E_{h}\right)_{i}=\varphi_{i}(0), \\
& \left(\Sigma_{h}\right)_{i, j}=\varphi_{i}(L) \varphi_{j}(L)
\end{aligned}
$$

Notice that $M_{h}^{L^{2}}, M_{h}^{H^{1}}, N_{h}^{L^{2}}(\omega), N_{h}^{H^{1}}(\omega)$ and $\Sigma_{h}$ are diagonal matrices, $B_{h}$ is block diagonal where the blocks are full and of size $r \times r+1$ and $E_{h}$ is a vector with only one non zero entry. This discrete formulation defines the following linear system on the global unknown $U_{h}$ :

$$
\begin{gathered}
A_{h}(\omega) U_{h}(\omega)=L_{h}, A_{h}(\omega)=\left(\begin{array}{l}
A_{11}(\omega) \\
A_{21}(\omega)
\end{array} A_{22}(\omega)\right. \\
L_{h}=\left(\begin{array}{c}
0 \\
E_{h}
\end{array}\right), \quad U_{h}(\omega)=\left(\begin{array}{c}
\hat{u}_{h} \\
\hat{p}_{h}
\end{array}\right)(\omega) \\
A_{11}(\omega)=j \omega M_{h}^{L^{2}}+j \omega N_{h}^{L^{2}}(\omega) \\
A_{12}(\omega)=-B_{h}, \quad A_{21}(\omega)=B_{h}^{*} \\
A_{22}(\omega)=j \omega M_{h}^{H^{1}}+j \omega N_{h}^{H^{1}}(\omega)+\frac{1}{Z_{R}(\omega)} \Sigma_{h}
\end{gathered}
$$

Notice that the matrix $A_{h}$ is sparse and can therefore be inverted by using efficient sparse routines

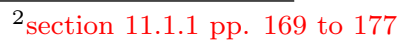

scipySparse ${ }^{3}$ Once this system is numerically solved, for a discrete set of values $\left\{\omega_{i}\right\}_{1 \leq i \leq N_{\omega}} \in$ $\left[\omega_{\min }, \omega_{\max }\right]$, the input impedance is

$$
\forall 1 \leq i \leq N_{\omega}, \quad Z_{\mathrm{FEM}}\left(\omega_{i}\right)=L_{h}^{*} U_{h}\left(\omega_{i}\right),
$$

which is the $\left(N_{L^{2}}+1\right)$ th term of the vector $U_{h}\left(\omega_{i}\right)$.

It is possible to diminish the computational burden by performing some pre-computations based on the pipe geometry and propagation hypotheses, and by taking advantage of the geometrical and arithmetical structure of the matrix $A_{h}$ and of the required output Amestoy et al.(2000), but this is out of the scope of the current article.

Finally, for a given frequency, the $N_{L^{2}}$ first terms of $U_{h}$ give an approximation of the velocity at every degree of freedom along the bore, while the $N_{H^{1}}$ last terms give an approximation of the pressure.

The FEM presented in this paper is implemented in OpenWind OpenWInD, an open source (GPLv3) Python 3 toolbox.

\section{Transfer matrix method}

The transfer matrix method (TMM) consists in writing relations between output and input acoustic variables of simple geometries (cylinders, cones, Bessel and exponential bores...) from the use of the propagation equations Caussé et al.(1984) Plitnik and Strong(1979). Consequently, given a radiation impedance $Z_{R}(\omega)$ and discretizing the bore profile in a series of $N_{p}$ parts, it is possible to compute the instrument's input impedance. Let $\left\{x_{i}\right\}_{0 \leq i \leq N_{p}}$ be the list of positions on the bore's axis defining all the parts (with $x_{0}=0$ and $x_{N_{p}}=L$ ). We also define $\hat{p}_{i}(\omega)$ and $\hat{u}_{i}(\omega)$ as approximations of the pressure and the volume flow calculated by the TMM at the positions $x_{i}$. When the TMM is exact, $\hat{p}_{i}(\omega)=\hat{p}\left(x_{i}, \omega\right)$ and $\hat{u}_{i}(\omega)=\hat{u}\left(x_{i}, \omega\right)$.

Formally, the relation between the input and the output of one part can be expressed as a $2 \times 2$ matrix $T_{i+1}(\omega)$ :

$$
\begin{aligned}
\left(\begin{array}{l}
\hat{p}_{i}(\omega) \\
\hat{u}_{i}(\omega)
\end{array}\right) & =\left(\begin{array}{ll}
a_{i+1}(\omega) & b_{i+1}(\omega) \\
c_{i+1}(\omega) & d_{i+1}(\omega)
\end{array}\right)\left(\begin{array}{l}
\hat{p}_{i+1}(\omega) \\
\hat{u}_{i+1}(\omega)
\end{array}\right) \\
& =T_{i+1}\left(\begin{array}{l}
\hat{p}_{i+1}(\omega) \\
\hat{u}_{i+1}(\omega)
\end{array}\right) .
\end{aligned}
$$

We then deduce the relation between the input and the output of the pipe:

$$
\zeta=\left(\begin{array}{c}
\hat{p}_{0}(\omega) / \hat{u}_{L}(\omega) \\
\hat{u}_{0}(\omega) / \hat{u}_{L}(\omega)
\end{array}\right)=\prod_{i=1}^{N_{p}} T_{i}(\omega)\left(\begin{array}{c}
Z_{R}(\omega) \\
1
\end{array}\right)
$$

${ }^{3}$ more precisely, scipy is linked to a BLAS (Basic Linear Algebra Subprogram) which depends on your operating system and what has been installed on the computer. All the results of this article have been computed using the BLAS/LAPACK intel MKL 2018 and the linear system resolutions use a SuperLU procedure. 
where $\hat{u}_{L}(\omega)$ is the volume flow at the pipe end, and finally $Z_{\mathrm{TMM}}=\frac{\zeta(1)}{\zeta(2)}$. The global transfer matrix is defined as the product of all the elementary matrices $T_{i}$. An implicit transmission condition is therefore assumed, which is the continuity of the variables between all parts. In practice, the computation is done only for a discrete set of pulsations $\left\{\omega_{j}\right\}_{1<j<N_{\omega}}$. In the sequel, we will only consider the TMM for cylinders and cones. Transfert matrices for other geometries are available in the literature $\operatorname{Braden}(2007)$ Chaigne and Kergomard(2016), Helie(2013)|.

For the lossless propagation case, the equations can be solved analytically for cones and cylinders and therefore the TMM provides the exact input impedance. In the presence of viscothermal losses, the dissipation terms depend non linearly on the bore radius, see Equation (2). It turns out that exact matrices can only be derived for the cylinder and not for more complex parts for which the radius depends on the space variable $\left(\hat{p}_{i}(\omega) \neq \hat{p}\left(x_{i}, \omega\right)\right)$. A first empirical approach handles this difficulty for conical parts by approximating them as a succession of cylinders of increasing or decreasing radii Caussé et al.(1984). A second empirical approach proposes to discretize each conical part in $N_{s u b}$ smaller cone subdivisions, and to use on each subdivision the transfer matrix derived for the cone considering lossless propagation, replacing some parameters by their lossy counterparts Chabassier and Tournemenne(2019) evaluated at a chosen intermediate radius $R^{\odot} \quad$ Mapes-Riordan(1993), $\left.\operatorname{Braden}(2007)\right]$. For a bore initially made of $N_{p}$ conical parts, the total number of actual transfer matrices to compute would be $N_{\text {TMM }}=N_{p} \times N_{\text {sub }}$.

Since the viscothermal losses depend non-linearly on the radius, no optimal value for $R^{\odot}$ can be immediately derived. Possible choices are the average radius $R^{\odot}=\left(R_{i}+R_{i+1}\right) / 2$ Mapes-Riordan(1993) (where $R_{i}$ and $R_{i+1}$ are the input and output radii of the cone subdivision), or any other weighted average Chaigne and Kergomard(2016), $\operatorname{Helie(2013).~In~}$ this article, we choose $R^{\odot}=\left(2 \min \left(R_{i}, R_{i+1}\right)+\right.$ $\left.\max \left(R_{i}, R_{i+1}\right)\right) / 3$, which seems to be used in some existing implementations of the TMM.

We show (see Chabassier and Tournemenne(2019) for more details) that using the TMM with the approximate matrix obtained with this strategy corresponds to actually solving analytically, for the approximated solutions $\check{u}$ and $\breve{p}$, the following system of equations:

$$
Z_{\mathrm{TMM}}(\omega)=\check{p}(0, \omega) \text {, where } \forall i \in\left[1, N_{\mathrm{TMM}}\right] \text {, }
$$

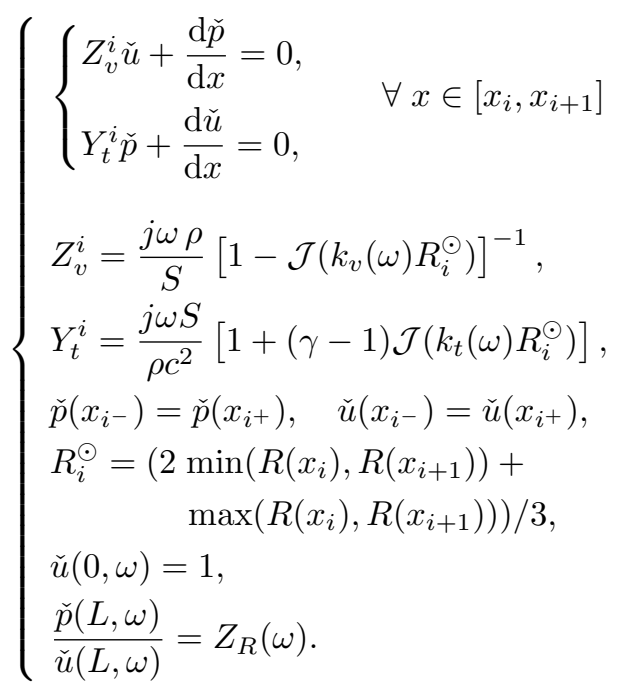

This problem is different from the continuous problem (7) solved with the FEM. The difference lies in the approximation $R^{\odot}$ inside the function $\mathcal{J}$ for every interval $\left[x_{i}, x_{i+1}\right]$ and amounts to approximating the original equation coefficients with discontinuous ones.

Finally, we propose a formulation unifying the transfer matrices of the cylinder and the cone, which coincides in either cases to the ones of the literature [Mapes-Riordan(1993), under visco-thermal losses. It reads:

$a_{i+1}(\omega)=a, b_{i+1}(\omega)=b, c_{i+1}(\omega)=c, d_{i+1}(\omega)=d$,

where

17) $\left\{\begin{aligned} a & =\frac{R_{i+1}}{R_{i}} \cosh \Gamma \ell-\frac{\beta}{\Gamma} \sinh \Gamma \ell \\ b & =\frac{R_{i}}{R_{i+1}} Z_{c} \sinh \Gamma \ell \\ c & =\frac{1}{Z_{c}}\left[\left(\frac{R_{i+1}}{R_{i}}-\frac{\beta^{2}}{\Gamma^{2}}\right) \sinh \Gamma \ell+\frac{\beta^{2} \ell}{\Gamma} \cosh \Gamma \ell\right] \\ d & =\frac{R_{i}}{R_{i+1}}\left(\cosh \Gamma \ell+\frac{\beta}{\Gamma} \sinh \Gamma \ell\right)\end{aligned}\right.$

where

$$
\begin{gathered}
\Gamma \equiv \Gamma\left(\omega, R^{\odot}\right)=\frac{j \omega}{c} \sqrt{\frac{1+(\gamma-1) \mathcal{J}\left(k_{t}(\omega) R_{i}^{\odot}\right)}{1-\mathcal{J}\left(k_{v}(\omega) R_{i}^{\odot}\right)}}, \\
Z_{c} \equiv Z_{c}\left(\omega, R^{\odot}\right)=\frac{\rho c}{S\left(x_{i}\right)} \sqrt{\frac{\left[1+(\gamma-1) \mathcal{J}\left(k_{t}(\omega) R_{i}^{\odot}\right)\right]^{-1}}{1-\mathcal{J}\left(k_{v}(\omega) R_{i}^{\odot}\right)}}
\end{gathered}
$$

and

$$
\beta=\frac{R_{i+1}-R_{i}}{\ell R_{i}},
$$

where $R_{i}$ and $R_{i+1}$ are respectively the input and output radii of the interval, $\ell$ is the axial length of the interval, and $R^{\odot}$ the previously defined quantity.

The transfer matrices for cylinders and cones in the lossless case can be similarly unified, it only requires to replace $\Gamma$ by $j \omega / c$ and $Z_{c}$ by $\rho c / S$.

The TMM presented in this paper is implemented in OpenWind OpenWInD. 


\section{Validation}

Unless otherwise stated, all input impedances presented hereafter are numerically computed from 20 to $2000 \mathrm{~Hz}$ with a $1 \mathrm{~Hz}$ step, the temperature is set to $25{ }^{\circ} \mathrm{C}$, and we consider a terminal impedance that models radiation from an infinite plane baffle Rabiner and Schafer(1978):

$$
Z_{R}(\omega)=\frac{\rho c}{S(L)} \frac{j \omega}{\alpha+j \omega \beta}
$$

where $\alpha=3 c \pi /(8 R)$ and $\beta=9 \pi^{2} / 128$. Other flanges can be modelled with this impedance form, by adjusting consequently the coefficients $\alpha$ and $\beta$, with a corresponding frequency validity range. Any other choice of radiation impedance can be done, including experimental ones, provided that the associated system of equations is well posed, meaning that its real part must be non-negative Chandler-Wilde(1997). The discussion about radiation impedances is out of the scope of this paper, but it is important to note that the following conclusions regarding convergence rates and accuracy do not depend on this choice.

In the following, the FEM meshes are constructed as follows. A target element size (TES) is chosen by the user. The instrument being described by a series of radii at different axial points, some of the instrument parts might be shorter than the TES, and some might be longer. The instrument parts longer than the TES are equally divided to only obtain elements smaller or equal to the TES. The instrument parts shorter than the TES are described by only one element having the same size than the part. For realistic instruments, any TES choice will produce a non-uniform mesh since the instrument parts are not necessarily commensurate. The ratio $\tau$ between the largest and smallest elements in a mesh is an indicator of this uniformity, and is equal to 1 for a uniform mesh.

Up to 8 geometries are studied in the following. One $20 \mathrm{~cm}$ cylinder with $5 \mathrm{~mm}$ radius (roughly corresponding to a trumpet leadpipe) is used to assess an error estimator for the lossy model. We use 5 different cones and one arbitrary simple discontinuous geometry to help analyze the TMM error for the lossy model. These geometries share their dimensions with existing instruments or instruments parts. They are intentionnally simple and have been selected in order be highly sensible to visco-thermal losses (small radius or fast slope). Besides, a trumpet-like bore based on measurements of a real commercial trumpet is used to provide a realistic study of the lossless and lossy models. Its bore is made of 9 cones to describe the mouthpiece, 4 cones for the leadpipe, 1 central cylinder and 20 cones for the bell (33 cones in total). Apart from the cylinder, the 7 other geometries are described in Figure 3. Notice that the 3 cones corresponding to the mouthpiece cup, backbore, and the trumpet leadpipe parts would normally be inside the instrument and yet we consider here their input impedance with open air radiation.

Notice that the relative errors that will be considered in the following of this paper are consequent to the discretization of the equations, and must be distinguished from the model error that would induce a discrepancy between the simulations and physical experiments. Quantifying this discretization error allows to correctly interpret the results of simulations.

All the results are obtained with OpenWind OpenWInD.

\subsection{Case without dissipation}

The TMM is numerically exact for the lossless model, and can therefore be taken as a reference in this case. Consequently, in order to assess the numerical quality of the FEM, we compute the relative error of the FEM solution to the reference solution obtained with the TMM, $E_{\mathrm{TMM}}$, in the lossless case, defined as:

$$
E_{\mathrm{TMM}}(i)=\frac{\left\|Z_{i \mathrm{FEM}}-Z_{\mathrm{TMM}}\right\|}{\left\|Z_{\mathrm{TMM}}\right\|},
$$

where $Z_{i \text { FEM }}$ is the impedance computed using the FEM at order $i$, and $Z_{\mathrm{TMM}}$ the impedance computed using the TMM, and $\|\cdot\|$ denotes the discrete $\ell^{2}$ norm of a vector over all the considered frequencies.

The upper part of Figure 4 shows the logarithm of $E_{\mathrm{TMM}}(i)$ with respect to the order $i$ of the FEM for the specific case of the trumpet bore displayed in Figure 3 .

The mesh is obtained by choosing a TES equal to $3.4 \mathrm{~cm}$, which gives $N=72$ elements, with a ratio $\tau=17$. We observe that the FEM provides a solution that is closer and closer to $Z_{T M M}$ as the order increases. After order 10 (which represents a total of 649 degrees of freedom for the $H^{1}$ variable, 1369 degrees of freedom in total), the impedance relative $\ell^{2}$ error does not diminish anymore and is close to $2.6 \mathrm{e}-$ 12 , which is dominated by roundup errors in double precision as expected. In the sequel we will call this a "converged solution". The linear convergence in logarithmic scale agrees with the finite elements theory which predicts an exponential order (spectral) convergence. The lower part of Figure 4 shows the logarithm of $E_{T M M}(i)$ with respect to the logarithm of the target element size (TES) of the mesh, for the different FEM orders 1 to 6 . Since the trumpet bore is composed of very large and very small parts, the observed curves are not yet exhibiting asymptotic rates of convergence (we would need much smaller TES in this case). However, we observe that for a given TES (and therefore mesh), increasing the order of the FEM always diminishes the relative $\ell^{2}$ error on the input impedance, achieving a precision that is difficult to reach by refining the mesh at a given order. 

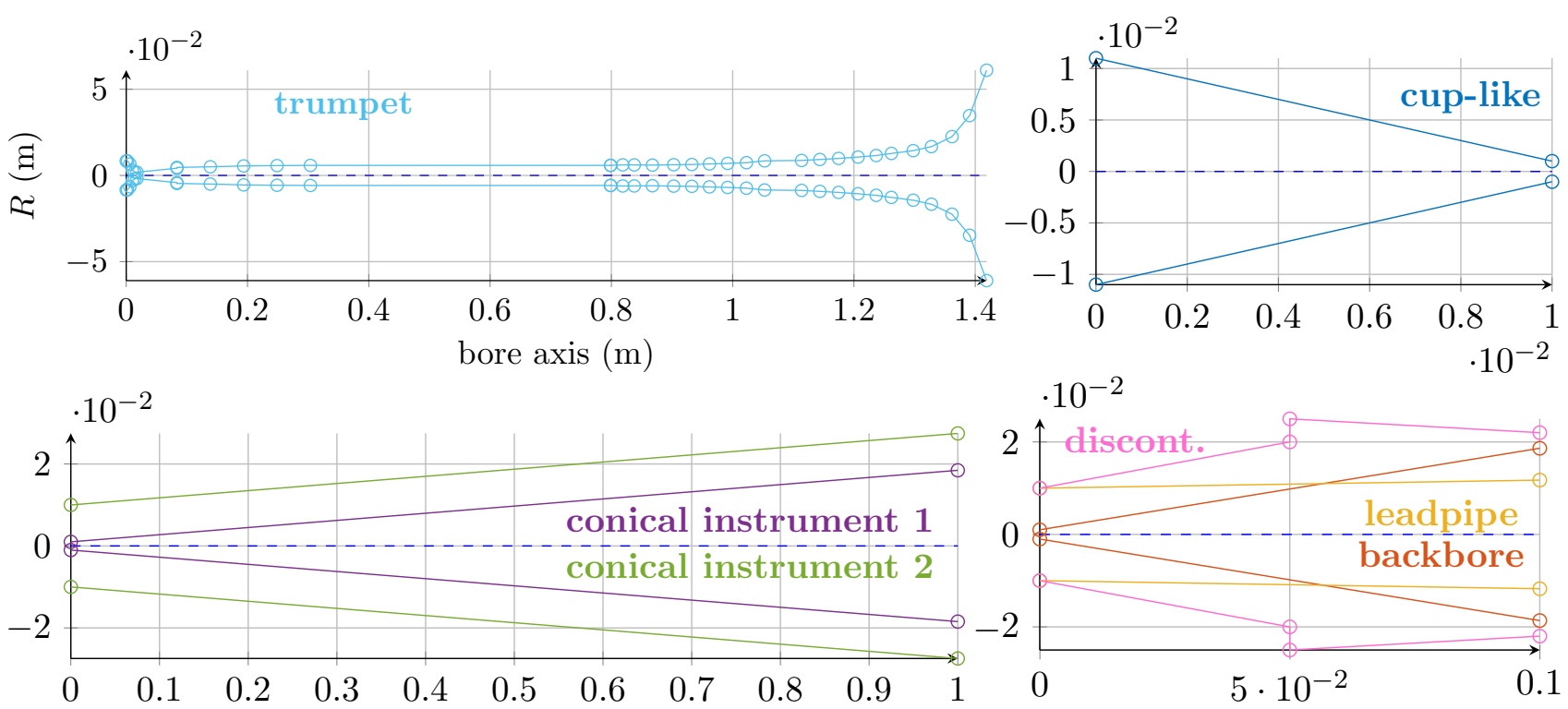

$\cdot 10^{-2}$

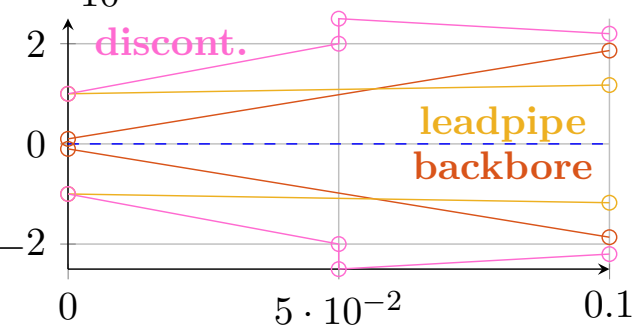

Figure 3: The seven studied bores. Top left : trumpet-like bore. Top right : simple convergent cone of general dimensions similar to a mouthpiece cup. Bottom left : two cones of $1 \mathrm{~m}$ representative of conical instruments. Bottom right : two cones being qualitatively similar to a mouthpiece backbore and a trumpet leadpipe part, and one arbitrary geometry made of two cones, one divergent, the other convergent, and a clear discontinuity between them. The circles represent the extremities of each part. (colors online)

\subsection{Case with dissipation}

Regarding the model with viscothermal losses (lossy model), the TMM is exact for cylinders only. It will thus not be possible to use $E_{\mathrm{TMM}}$ to assess FEM convergence towards the exact solution for geometries of arbitrary shapes. Instead, we compute the relative $\ell^{2}$ error between two finite element computations on the same mesh but consecutive orders:

$$
E_{\text {order }}(i)=\frac{\left\|Z_{i+1 \text { FEM }}-Z_{i \text { FEM }}\right\|}{\left\|Z_{i \text { FEM }}\right\|},
$$

which is a heuristic and customary estimator when no exact solution is available (attributed to $\mathrm{C}$. Runge, see $\operatorname{Repin(2008)]})$. Notice that it is not a mathematical a posteriori estimator Babuska(1981). Ainsworth(1997) but must be considered only as an illustration.

\begin{tabular}{llllll} 
order & 1 & 2 & 3 & 4 & $5+$ \\
\hline $\begin{array}{l}\text { frequential } \\
\text { deviation (cents) }\end{array}$ & 236 & 26 & 0.3 & 0.01 & $<1 \mathrm{e}-4$ \\
$\begin{array}{l}\text { amplitude } \\
\text { deviation (dB) }\end{array}$ & 15 & 1.8 & 0.02 & 0.001 & $<1 \mathrm{e}-5$
\end{tabular}

Table 2: Frequential position and amplitude deviations of the second impedance peak of the $20 \mathrm{~cm}$ cylinder (radius $5 \mathrm{~mm}$ ) using the lossy model. The reference is computed using the TMM. A visual representation of this second peak is shown Figure6.

The first considered case is a cylinder $20 \mathrm{~cm}$ long with a $5 \mathrm{~mm}$ radius, which could be compared qualitatively to a trumpet leadpipe in terms of dimensions.
In Figure 5, we consider a mesh of $N=3$ elements and we represent both the $E_{\mathrm{TMM}}$ and the $E_{\text {order }}$ relative $\ell^{2}$ error estimators, since $E_{\mathrm{TMM}}$ is relevant in this case (it measures the distance to an exact solution). The two error estimators exhibit a very similar behavior which illustrates the fact that they are both relevant to assess the convergence of the FEM. In this case, the FEM provides a converged solution at order 9. The fact that $E_{\text {order }}$ tends to machine precision illustrates the usual finite elements convergence theory Fortin (1977), Cohen (2000) which theoretically ensures that the obtained numerical solution is actually close to the exact impedance of the considered instrument (as opposed to a converged but false numerical solution) Dauge et al.(2005).

Figure 6 shows the modulus of the input impedance computation for the same cylinder with respect to the frequency, for different FEM orders. Table 2 gives the frequential and amplitude deviations of the second peak. The difference between the curves is visible for all orders, which is consistent with the fact that the solution is not yet converged. At a given order, the error increases with the frequency, which is known as the "pollution effect" Gerdes and Ihlenburg(1999). When the order increases, the solution becomes valid in a wider frequency range. Two main effects are to be noted in the context of musical acoustics: the peaks amplitudes and frequencies can be wrong, the latter being due to numerical dispersion Ihlenburg and Babuška(1995). Increasing the number of elements and/or the order allow to reduce these effects down to machine precision. In this case, at low 


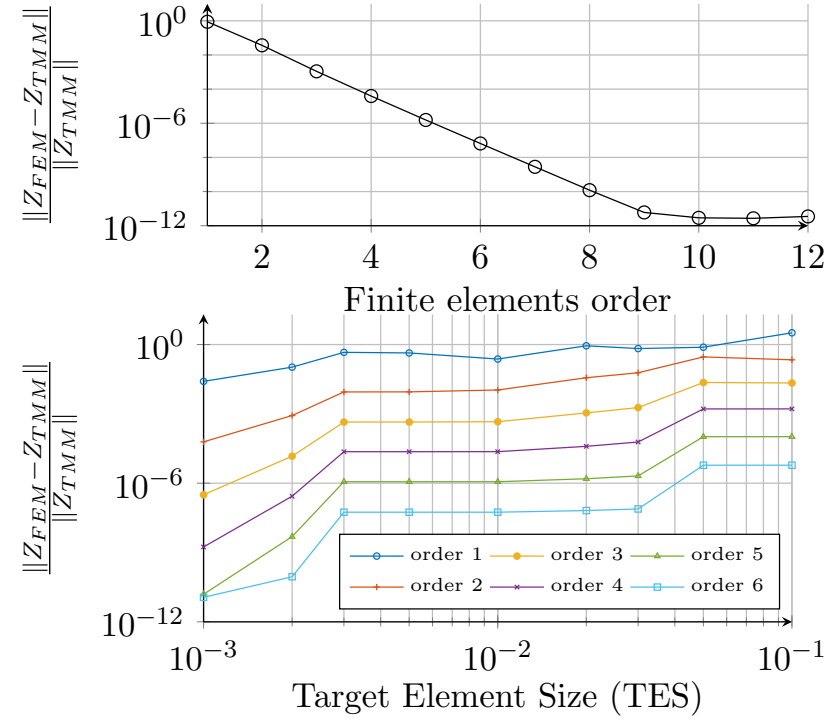

Figure 4: Relative $\ell^{2}$ error between the input impedance obtained with the FEM and the TMM for the trumpet under lossless conditions. Top: the finite elements order varies on a given mesh, Bottom: the target element size (TES) varies for different FEM orders. (colors online)
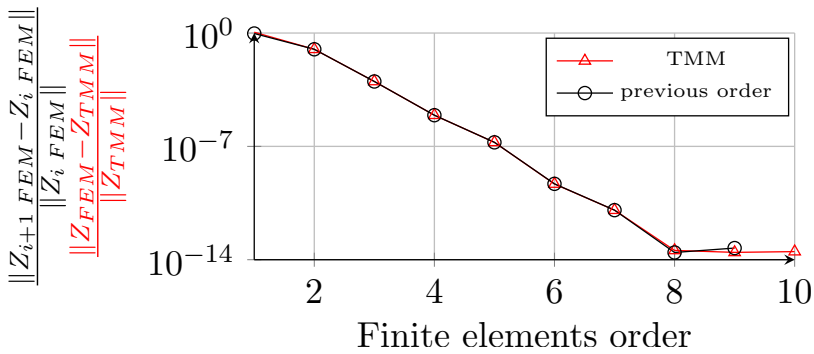

Figure 5: Comparison between $E_{\text {order }}$ and $E_{\mathrm{TMM}}$ for a $20 \mathrm{~cm}$ cylinder of radius $5 \mathrm{~mm}$ using the lossy model. The FEM mesh is uniform with 3 elements.

orders of discretization, erroneous conclusions can be drawn if the user does not attribute the dispersion to the numerical approximation but to the model.

$$
\text { Notice finally that finite differences }
$$
Bilbao and Chick(2013)] can be seen, at least locally, as first order finite elements. The analyses of Figures 4 and 6 illustrate the fact that using a first order approximation can be a source of inaccuracy in the context of musical acoustics.

Figure 7 shows the logarithm of the consecutive relative $\ell^{2}$ error $E_{\text {order }}$ with respect to the FEM order, considering the geometries of Figure 3, in the lossy case. The number of elements is indicated in the legend. An exponential order convergence is still observed in the presence of dissipation which is in agreement with the FEM theory since only the coefficients have changed. Depending on the case, the solution seems to be converged at an order ranging between 5 and 10 , which is related to the properties of the cho-

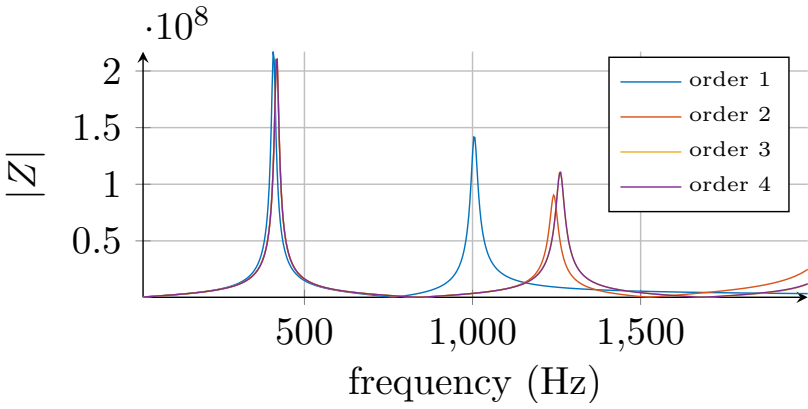

Figure 6: Modulus of the input impedance of a 20 $\mathrm{cm}$ cylinder of radius $5 \mathrm{~mm}$ computed by the FEM at different orders. (colors online)

sen mesh and to mathematical constants depending on the exact solution.
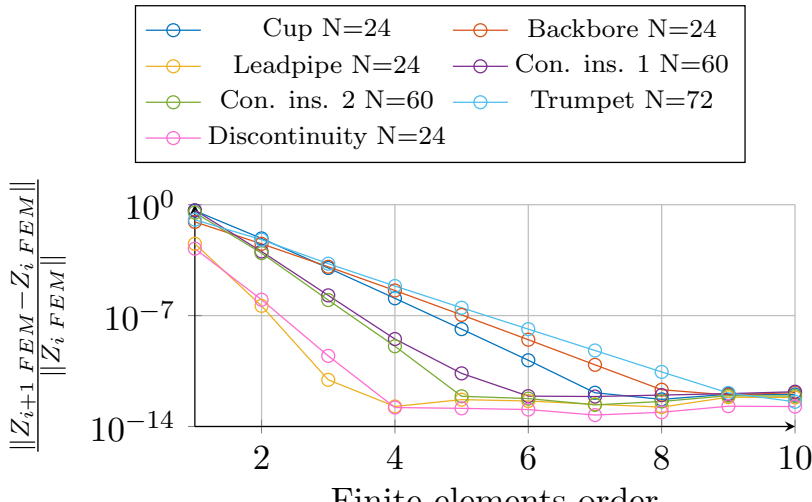

Figure 7: Consecutive relative $\ell^{2}$ error between the input impedances obtained with the FEM for the lossy model using the bores of Figure 3 with respect to the FEM order. The number of elements of each mesh is given in the legend for each geometry. (colors online, matching with Figure 3 .

\section{Results}

\subsection{Study of the TMM error for arbi- trary shapes considering losses}

Given the results of the previous sections, a converged FEM solution can therefore be considered as the reference numerical solution for the lossy model, on geometries for which no exact solution is available. As said earlier, the TMM used on the lossy model is not exact for bores of arbitrary shape, and follows an empirical approach to compute input impedances, see section 4. In this study, we investigate the second empirical approach, subdividing every conical part in $N_{\text {sub }}$ equal segments and using for each subdivision the formula (17), which amounts to solving the approximate Equations (16).

It is possible to study the error made by the TMM 
approximation, by computing the relative $\ell^{2}$ error with the converged FEM input impedance:

$$
E_{\text {conv FEM }}(j)=\frac{\left\|Z_{j \mathrm{TMM}}-Z_{\text {conv FEM }}\right\|}{\left\|Z_{\text {conv FEM }}\right\|},
$$

where $Z_{j \text { TMM }}$ is the input impedance computed using the TMM with $j$ subdivisions for each instrument part, and $Z_{\text {conv FEM }}$ is the converged impedance obtained by the FEM.

Since both methods solve different systems of equations (namely, Equations (7) for the FEM and Equations (16) for the TMM), the error between their solutions will be related to the difference between their equations Chabassier and Tournemenne(2019)]. As $j$ increases, the TMM equations tend to the FEM equations and thus we expect both solutions to converge.

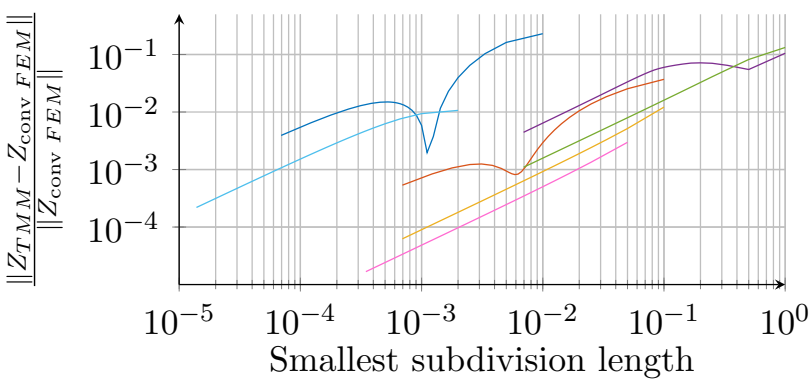

Figure 8: Relative $\ell^{2}$ error between TMM solution and the converged FEM solution for the bores of Figure 3, w.r.t. the smallest subdivision length used for the TMM computation. (colors online, matching with Figure 3p

Figure 8 shows the logarithm of $E_{\text {conv FEM }}$ with respect to the logarithm of the smallest subdivision length $\Delta x_{j}$ used to compute $Z_{j}$ TMM, for the different bores displayed in Figure 3 . The relative error is computed on a frequency range of $[20,2000] \mathrm{Hz}$ with a $1 \mathrm{~Hz}$ step, but the obtained results are similar when a different frequency range is considered. A first observation is that all curves are decreasing at rate close to 1 asymptotically (error divided by 10 when the subdivisions length is divided by 10). For the first conical instrument, the mouthpiece backbore and more extensively, for the cup-like bore, the curves show a dip for a specific subdivision length value. This can happen when considering few subdivisions for each cone and disappears asymptotically, and can be interpreted as fortuitous values of $R^{\odot}$ for the cones subdivisions. More quantitatively, the error $E_{\text {conv FEM }}$ illustrates the difference between the discretized TMM approach problem (16) and the original system (7). Because the convergence is slow (order 1 w.r.t. the subdivision length), the number of TMM subdivisions needed to obtain a solution that has converged up to machine precision is very large and induces a very heavy computational cost.

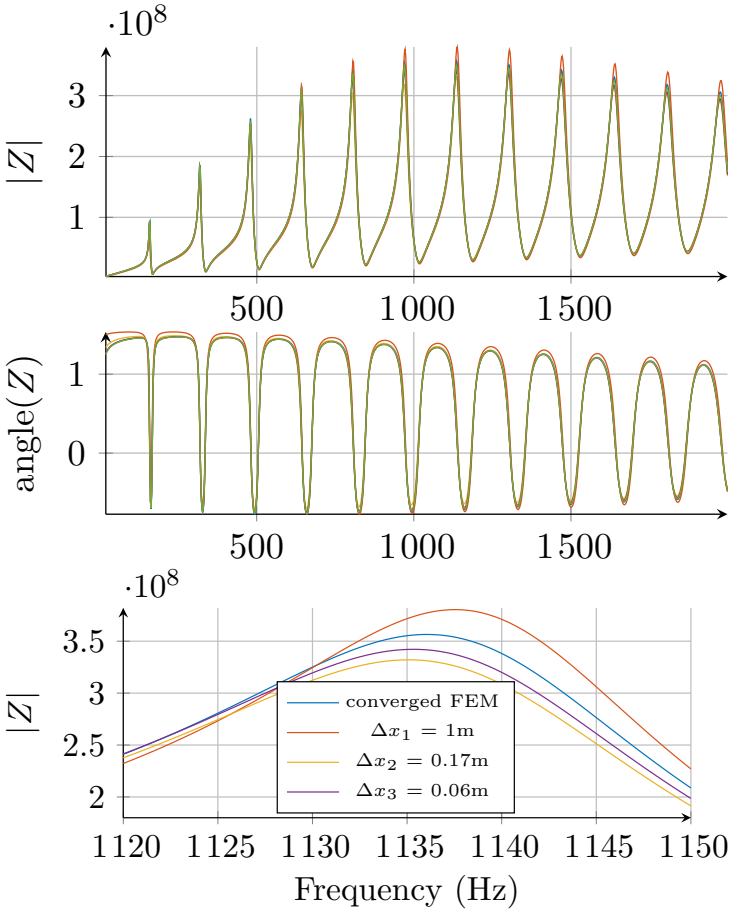

Figure 9: Impedance comparison between the converged FEM and the TMM method using different subdivision lengths of the Conical instrument 1. (colors online)

Figure 9 shows the input impedance of the instrument Conical inst. 1 on the frequency range $[0,2]$ $\mathrm{kHz}$ and $[1120,1150] \mathrm{Hz}$ (close to the 7 th impedance peak). On this example, the amplitude and frequency position of the impedance peaks are misjudged by the TMM when the number of subdivisions is too low. For example, the height of the 7th peak of this instrument is $6.9 \%$ too low $(3.56 \mathrm{e} 8$ against $3.32 \mathrm{e} 8$ ) when considering a subdivision length of $0.17 \mathrm{~m}$ (6 subdivisions), and its frequency position is 1.37 cents too low $(1136 \mathrm{~Hz}$ against $1135 \mathrm{~Hz})$. In the case of the cup-like bore, this frequency shift is even higher (4.99 cents for the first peak around $2000 \mathrm{~Hz}$ with a subdivision length of $0.01 \mathrm{~m}$ (1 subdivision) for the TMM).

\subsection{Computation time and features comparison of the two approaches}

Computation time In the previous paragraphs, we have seen that both the FEM and the TMM are relevant to compute the input impedance of a given instrument as defined in Equations (7). In order to complete the methods' performance analysis, it is necessary to assess and compare their computational costs. Fast input impedance computation is especially useful when considering optimisation applications where a large number of input impedances must be computed to reach optimal designs. Recall that the FEM computation requires the inversion of the sparse linear system (10) while the TMM computation requires 
the evaluation of the matrices product (14), both for a discrete set of pulsations $\left\{\omega_{i}\right\}_{1 \leq i \leq N_{\omega}}$. In the case of the FEM, most of the computation time is spent in computing the finite element matrices $(10 \%)$, inverting them (39\%), and evaluating the dissipative terms if any (48\%) (these percentages depend somehow on the number of degrees of freedom). The matrices to invert are sparse and the overall conditioning of the matrices is good thanks to the use of spectral high order finite elements. A fair comparison can only be performed for numerical solutions that provide the same precision with respect to the exact solution. Since the FEM relies on the choice of both a mesh and an order, the same precision can be obtained with several situations that do not necessarily induce the same computational cost. In the sequel, the given time is always the smallest manually found computational time.

Firstly, for the cases where the TMM are exact (lossless case, lossy cylinder), the TMM computation is very competitive and provides the exact solution with only roundup errors. On the contrary, the FEM needs to be converged in order to provide a solution with a similar precision, and this induces an extra computational cost (about 1883 times more for the lossless trumpet and 194 times more for the lossy cylinder).

In the presence of viscothermal losses and arbitrary shapes, the TMM is not exact anymore and uses a discrete and empirical approach to compute the input impedance. We display in Figure 10 the computation times with respect to the relative $\ell^{2}$ error to the converged solution, for the realistic trumpet-like bor ${ }^{4}$. for several TMM subdivision lengths (from $\Delta x=2 \mathrm{e}-$ $3 \mathrm{~m}$ to $1.3 \mathrm{e}-5 \mathrm{~m})$ and for the FEM with 35 elements at order 4.

Finally, Another FEM strategy called "adaptative" is also considered: it adapts the order of each mesh element to its size. This strategy avoids introducing too many degrees of freedom in small elements, improving the computation time without diminishing the global $\ell^{2}$ error. In the specific case of the trumpet-like bore with a TES (Target Element Size) producing 35 elements, the first parts describing the mouthpiece are few millimeters long which is shorter than the TES. Consequently, the 4 interpolation points are unnecessarily cramped up on the only element of each of these parts. Therefore, a manual definition of the best order for each element, aided by the expected local shortest wavelength, is undertaken in order to obtain a good compromise between the number of degrees of freedom and the precision. In the example of Figure 10. the adaptative FEM improves the computation time by $11.1 \%$ compared with the usual FEM, and both computations lead to a relative $\ell^{2}$ error of $4.1 \times 10^{-4}$.

The fastest TMM setting $(\Delta x=2 \mathrm{e}-3 \mathrm{~m})$, provides a

\footnotetext{
${ }^{4}$ Computations run on a $3.4 \mathrm{GHz}$ Intel Core i7-2600 with 16 GB of RAM
}

relative $\ell^{2}$ error equal to $1.1 \%$ and computes the input impedance in 0.225 seconds, which is 11.2 times faster than the adaptative FEM (2.5 seconds). The most precise TMM setting has a precision similar to the FEM $\left(2.2 \times 10^{-4}\right)$, but the computation time is 11.9 times higher than the adaptative FEM (30.1s). Other orders (2,3 and 5 ) have been considered for the mesh of 35 elements. Corresponding results are listed in Table 3 and the order 3 is displayed on Figure 10 All the computation times are similar (between 2 and 3.2 seconds) while the errors greatly improve (from $8.8 \mathrm{e}-2$ to 2.5e-5). This shows the overall numerical performance of the FEM in real life situations, which can target a specific precision while maintaining a competitive computation time.

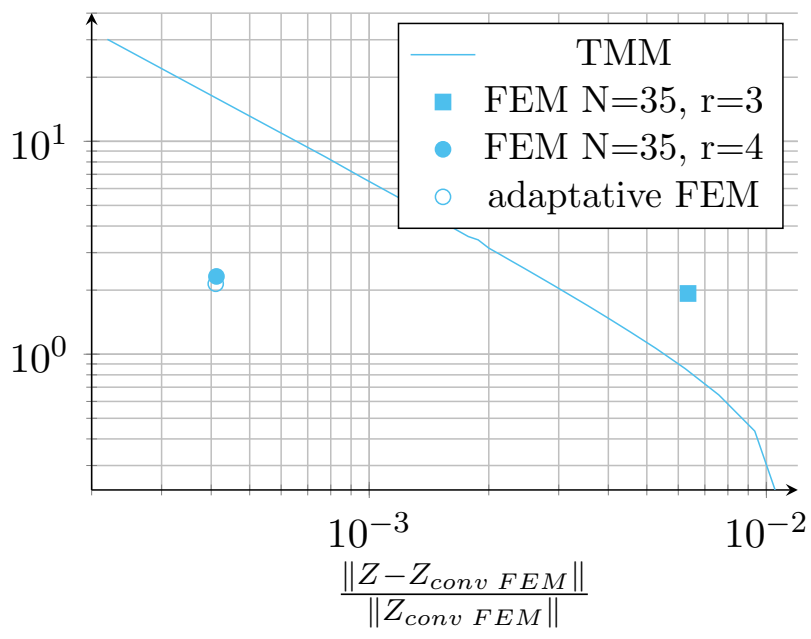

Figure 10: CPU time of the trumpet impedance computation w.r.t impedance relative $\ell^{2}$ error. Comparison between the TMM and FEM methods.

\begin{tabular}{ccccc} 
elements \# & 35 & 35 & 35 & 35 \\
$\begin{array}{c}\text { order } \\
\text { degrees of } \\
\text { freedom }\end{array}$ & 2 & 3 & 4 & 5 \\
\hline CPU time (s) & 2 & 140 & 175 & 210 \\
$E_{\text {conv FEM }}$ & $8.8 \mathrm{e}-2$ & $6.3 \mathrm{e}-3$ & $4.1 \mathrm{e}-4$ & $2.5 \mathrm{e}-5$
\end{tabular}

Table 3: Different computation times and $E_{\text {conv FEM }}$ considering different orders for the trumpet impedance using a 35 elements discretization.

Acoustic variables One immediate feature permitted by the FEM is the availability of the pressure and volume flow spectra along the entire bore axis, see Figure 11, which is directly obtained by considering all the vector $U_{h}$ of system (10) (and not only the term corresponding to the input pressure). This output therefore comes at no extra computational cost compared to the impedance computation. Interpolation on arbitrary points is also possible without increasing the numerical error. 
It could also be possible to reconstruct the pressure and volume flow using the TMM, but it would induce extra computational cost due to either over sampling of the bore profile (storing intermediate results of matrix products) or value interpolation (for which an arbitrary interpolation rule must be chosen and could potentially deteriorate the numerical result).

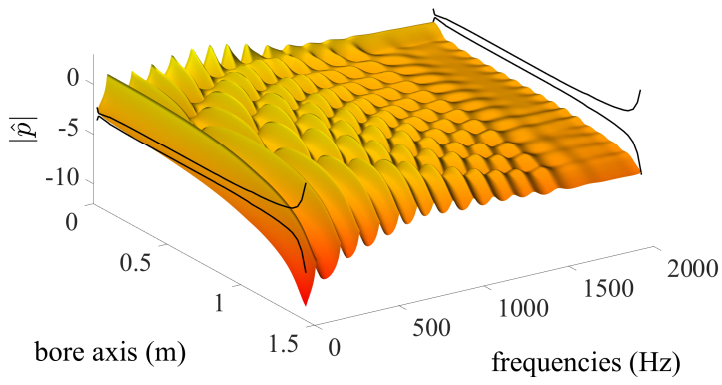

Figure 11: Evolution of the pressure modulus in logarithmic scale along the bore of the lossy trumpet according to frequency. The border at the beginning of the instrument (bore axis $x=0$ ) displays the input impedance. (colors online)

In the case of a wind instrument, it helps to understand where the nodes and antinodes of the waves are located, which may help instrument makers better visualize the instrument's functioning or even position the tonehole:5

Extended physical situations One major advantage of using FEM over TMM is the possibility to easily solve equations with no available analytical solution while maintaining an arbitrary precision. Indeed, when more complex cases than lossless acoustic propagation are considered, it may be impossible to find analytical solutions, requiring the TMM to consider some approximations if possible (viscothermal losses, continuously non-constant physical coefficients). This feature could potentially give access to instruments impedances in very interesting physical situations. For instance, it is theoretically and technically straightforward to consider non-constant physical coefficients, as in the case where the temperature varies inside the pipe. Indeed, this only prompts different values for the matrices $M_{h}^{L^{2}}, M_{h}^{H^{1}}, N_{h}^{L^{2}}(\omega)$ and $N_{h}^{H^{1}}(\omega)$. Using exactly the same quadrature formulae, this only results in a different integrand taking into account the temperature value throughout the bore axis. The TMM can achieve a similar goal with less flexibility and less control on the discretisation error, refining the bore parts definition and considering a different constant temperature on each refined parts.

\footnotetext{
${ }^{5}$ private discussion with the instrument maker Augustin
} Humeau
Table 4 shows the frequential position and amplitude deviations of the 9 first impedance peaks of the trumpet between a linear temperature gradient Gilbert et al.(2006) between 37 and $21{ }^{\circ} \mathrm{C}$, and an averaged temperature of $29^{\circ} \mathrm{C}$ inside the bore. There is a $7 \%$ difference between the two moduli of the impedances, showing the importance of the temperature gradient for impedance calculation. More precisely, the frequential deviation varies between 0.3 and 4.2 cents ( 1.9 cents in average), and the peaks amplitude varies between 0.1 and $0.3 \mathrm{~dB}$.

\begin{tabular}{llllllllll} 
peak \# & 1 & 2 & 3 & 4 & 5 & 6 & 7 & 8 & 9 \\
\hline $\begin{array}{l}\text { frequential } \\
\text { deviation (cents) }\end{array}$ & 4.2 & 1.5 & 1.4 & 1.7 & 2.6 & 1.5 & 1.4 & 2.1 & 0.3 \\
$\begin{array}{l}\text { amplitude } \\
\text { deviation (dB) }\end{array}$ & 0.1 & 0.2 & 0.2 & 0.3 & 0.3 & 0.2 & 0.2 & 0.2 & 0.1
\end{tabular}

Table 4: Frequential position and amplitude deviations between the two temperature profiles along the bore of the trumped for the lossy model, for the 9 first impedance peaks.

Other possibilities include the accurate consideration of arbitrary bores (Bessel, exponential, polynomials, splines, ...), the possible integration of new terms in the equations or the coupling with other equations modelling different physical phenomena (pipes junctions, or excitators as lips, reeds, flue, ...).

\section{Conclusion and prospects}

The precision and performances of FEM and TMM have been assessed, based on quantitative comparison, as well as the exhibition of the actual equation solved by the TMM. In realistic cases as a trumpet with losses, the FEM allows to compute the same numerical solution as the TMM with a limited computational cost. It also allows to compute unusual physical situations as non-constant coefficients along the bore. Moreover, the computation gives a direct access to the acoustic variables inside the pipe for no extra computational cost or over-sampling. All the results of this article have been computed and can be run again using the open-source python toolbox OpenWind OpenWInD. Two direct extensions can follow this work: the implementation of toneholes in the model in order to model the input impedance of woodwind instruments, and the sound synthesis based on the same finite element method in space and finite difference in time. Notice that the presence of viscothermal terms induces a major theoretical difficulty in the time domain Berjamin et al.(2017). Finally this finite element framework is an efficient basis aiming at developing an inversion algorithm based on the fullwaveform inversion Virieux and Operto(2009)]. This technique can be used to optimize the instrument's geometry based on criteria derived from the input impedance, and relies strongly on the additional out- 
puts of the FEM impedance computation which are the pressure and flow fields inside the instrument.

\section{References}

[scipySparse] 'https://docs.scipy.org/doc/ scipy/reference/sparse.html'.

[OpenWInD] 'https://gitlab.inria.fr/ openwind/release under GPLv3 licence Chabassier, J., Tournemenne, R." .

[Ainsworth(1997)] Ainsworth, M. and Oden, J. T. (1997). "A posteriori error estimation in finite element anaysis," Comput. Methods in Appl. Mech. Eng. 142, 1-88.

[Amestoy et al.(2000)] Amestoy, P. R., Duff, I., and L'Excellent, J.-Y. (2000). "Multifrontal parallel distributed symmetric and unsymmetric solvers," Comput. Methods in Appl. Mech. Eng. 184, 501-520.

[Babuska(1981)] Babuska, I. and Dorr, M. R. (1981). "Error Estimates for the combined $\mathrm{h}$ and $\mathrm{p}$ versions of the finite element method.," Numer. Math. 37, 257-277.

[Backus(1976)] Backus, J. (1976). "Input impedance curves for the brass instruments," The Journal of the Acoustical Society of America 60(2), 470480 .

[Berjamin et al.(2017)] Berjamin, H., Lombard, B., Vergez, C., and Cottanceau, E. (2017). "TimeDomain numerical modeling of brass instruments including nonlinear wave propagation, viscothermal losses, and lips vibration," Acta Acust united Ac 103(1), 117-131.

[Bilbao(2009)] Bilbao, S. (2009). "Direct simulation of reed wind instruments," Computer Music Journal 33(4), 43-55.

[Bilbao and Chick(2013)] Bilbao, S., and Chick, J. (2013). "Finite difference time domain simulation for the brass instrument bore," J. Acoust. Soc. Am. 134(5), 3860-3871.

[Braden(2007)] Braden, A. C. P. (2007). "Bore optimisation and impedance modelling of brass musical instruments," Ph.D. thesis, University of Edinburgh.

[Braden et al.(2009)] Braden, A. C. P., Newton, M. J., and Campbell, D. M. (2009). "Trombone bore optimization based on input impedance targets," J. Acoust. Soc. Am. 125(4), 2404-2412.

[Brezis(2011)] Brezis, H. (2011). "Functional analysis, Sobolev spaces and partial differential equations," (Springer New York, London).
[Buys et al.(2017)] Buys, K., Sharp, D., and Laney, R. (2017). "Developing and evaluating a hybrid wind instrument," Acta Acust united Ac 103(5), 830-846.

[Campbell(2004)] Campbell, M. (2004). "Brass instruments as we know them today," Acta Acust united Ac 90(4), 600-610.

[Caussé et al.(1984)] Caussé, R., Kergomard, J., and Lurton, X. (1984). "Input impedance of brass musical instruments - comparison between experiment and numerical models," J. Acoust. Soc. Am. 75(1), 241-254.

[Chabassier and Tournemenne(2019)] Chabassier, J., and Tournemenne, R. (2019). "About the transfert matrix method in the context of acoustical wave propagation in wind instruments," INRIA Research Report 9254.

[Chaigne and Kergomard(2016)] Chaigne, A., and Kergomard, J. (2016). Modern Acoustics and Signal Processing "Acoustics of Musical Instruments:," (Springer New York).

[Chandler-Wilde(1997)] Chandler-Wilde, S. N. (1997). "The impedance boundary value problem for the Helmholtz equation in a half-plane," Mathematical Methods in the Applied Sciences 20, 813-840.

[Cohen(2004)] Cohen, G. (2004). "Higher Order Numerical Methods for Transient Wave Equations," (Springer, Berlin, Heidelberg).

[Cohen (2000)] Cohen, G. and Fauqueux, S. (2000). "Mixed finite elements with mass-lumping for the transient wave equation," Journal of Computational Acoustics 8 (1), 171-188.

[Courant and Hilbert(1965)] Courant, R., and Hilbert, D. (1965). "Methods of mathematical physics. partial differential equations," Interscience $\mathbf{2}$.

[Dalmont et al.(2001)] Dalmont, J.-P., Nederveen, C. J., and Joly, N. (2001). "Radiation impedance of tubes with different flanges: Numerical and experimental investigations," Journal of Sound and Vibration 244(3), 505 - 534 .

[Dauge et al.(2005)] Dauge, M., Costabel, M., and Schwab, C. (2005). "Exponential convergence of hp-fem for maxwell's equations with weighted regularization in polygonal domains," Math. Models Methods Appl. Sci. 15(4), 575-622.

[Fortin (1977)] Fortin, M. (1977). "An analysis of the convergence of mixed finite element methods," RAIRO. Analyse Numérique 11 (4), 341-354. 
[Gerdes and Ihlenburg(1999)] Gerdes, K., and Ihlenburg, F. (1999). "On the pollution effect in FE solutions of the 3D-Helmholtz equation," Computer Methods in Applied Mechanics and Engineering 170(1-2), 155-172.

[Gilbert et al.(2006)] Gilbert, J., Ruiz, L. L., and Gougeon, S. (2006). "Influence de la température sur la justesse d'un instrument à vent," in Proceedings of Congres Français d'Acoustique 2006, Tours.

[Giordano(2014)] Giordano, N. (2014). "Simulation studies of a recorder in three dimensions," J. Acoust. Soc. Am. 135(2), 906-916.

[Helie(2013)] Hélie, Thomas and Hézard, Thomas and Mignot, Rémi and Matignon, Denis (2013). "One-dimensional acoustic models of horns and comparison with measurements," Acta Acust united Ac 99(6), 960-974.

[Ihlenburg and Babuška(1995)] Ihlenburg, F., and Babuška, I. (1995). "Dispersion analysis and error estimation of galerkin finite element methods for the Helmholtz equation," Int. J. Numer. Methods Engrg. 38, 3745-3774.

[Kausel(2001)] Kausel, W. (2001). "Optimization of brasswind instruments and its application in bore reconstruction," Journal of New Music Research 30(1), 69-82.

[Kirchhoff(1868)] Kirchhoff, G. (1868). "Ueber den einfluss der wärmeleitung in einem gase auf die schallbewegung," Annalen der Physik 210(6), 177-193.

[Le Roux et al.(2008)] Le Roux, J. C., Dalmont, J.P., and Gazengel, B. (2008). "A new impedance tube for large frequency band measurement of absorbing materials," J. Acoust. Soc. Am. $\mathbf{1 2 3}(5), 3119$.

[Lefebvre and Scavone(2012)] Lefebvre, A., and Scavone, G. P. (2012). "Characterization of woodwind instrument toneholes with the finite element method," The Journal of the Acoustical Society of America 131(4), 3153-3163.

[Mapes-Riordan(1993)] Mapes-Riordan, D. (1993). "Horn modeling with conical and cylindrical transmission-line elements," J. Audio Eng. Soc. 41(6), 471-484.

[Plitnik and Strong(1979)] Plitnik, G. R., and Strong, W. J. (1979). "Numerical method for calculating input impedances of the oboe," The Journal of the Acoustical Society of America 65(3), 816-825.
[Quarteroni et al.(2007)] Quarteroni, A., Sacco, R., and Saleri, F. (2007). "Méthodes Numériques : Algorithmes, analyse et applications," (SpringerVerlag Mailand).

[Rabiner and Schafer(1978)] Rabiner, L. R., and Schafer, R. W. (1978). "Digital processing of speech signals,", 100 (Prentice-hall Englewood Cliffs, NJ).

[Repin(2008)] Repin, S. (2008). "A Posteriori Estimates for Partial Differential Equations ,", Radon Series on Computational and Applied Mathematics (Berlin, Boston: De Gruyter).

[Rienstra(2005)] Rienstra, S. W. (2005). "Webster's horn equation revisited," SIAM Journal on Applied Mathematics 65(6), 1981-2004.

[Sharp et al.(2011)] Sharp, D., Mamou-Mani, A., and van Walstijn, M. (2011). "A single microphone capillary-based system for measuring the complex input impedance of musical wind instruments," Acta Acust united Ac 97(5), 819-829.

[Silva et al.(2014)] Silva, F., Vergez, C., Guillemain, P., Kergomard, J., and Debut, V. (2014). "MoReeSC: A framework for the simulation and analysis of sound production in reed and brass instruments," Acta Acust united Ac 100(1), 126138.

[Tournemenne et al.(2017)] Tournemenne, R., ${ }_{885}$ Petiot, J.-F., Talgorn, B., Kokkolaras, M., and Gilbert, J. (2017). "Brass instruments design using physics-based sound simulation models and surrogate-assisted derivative-free optimization," Journal of Mechanical Design 139(4), 041401.

[van den Doel and Ascher(2008)] van den Doel, K., and Ascher, U. M. (2008). "Real-time numerical solution of webster's equation on a nonuniform grid," IEEE Transactions on Audio, Speech, and Language Processing 16(6), 1163-1172, doi: 10.1109/TASL . 2008.2001107

[Virieux and Operto(2009)] Virieux, J., and Operto, S. (2009). "An overview of full-waveform inversion in exploration geophysics," Geophysics 74(6), WCC1-WCC26.

[Webster(1947)] Webster, J. C. (1947). "An electrical method of measuring the intonation of cupmouthpiece instruments," The Journal of the Acoustical Society of America 19(5), 902-906.

[Zwikker and Kosten(1949)] Zwikker, C., and Kosten, C. W. (1949). "Sound absorbing materials," (Elsevier). 